\title{
PLANEACIÓN ESTRATÉGICA: CASO DE ESTUDIO APLICADO A UNA FÁBRICA DE LADRILLOS EN COLOMBIA.
}

\section{Strategic Planning: Case study applied to a brick factory in Colombia}

\begin{tabular}{|c|c|}
\hline Julián Camilo Rey Sánchez (D) & Yolanda Sepúlveda Mujica (D) \\
\hline Beyker Aldair García Delgado (D) & Sebastián García Méndez (D) \\
\hline $\begin{array}{r}\text { Estudiante Ingeniería Industrial, Universidad de } \\
\text { Investigación y Desarrollo (Bucaramanga, Colombia). } \\
\text { Correo electrónico: irey9@udi.edu.co } \\
\text { bgarcia2@udi.edu.co }\end{array}$ & $\begin{array}{l}\text { Magíster en Administración, docente de la Universidad de } \\
\text { Investigación y Desarrollo (Bucaramanga, Colombia). } \\
\text { Correo electrónico: ysepulveda1@udi.edu.co } \\
\text { sgarcia15@@udi.edu.co }\end{array}$ \\
\hline
\end{tabular}

| Recibido/Received: 13/01/2021 • Aprobado/Approved: 29/06/2021 • Publicado/Published: 30/07/2021

\section{Resumen}

En el presente trabajo se analizan los factores de cambio en el desarrollo de estrategias, en una empresa de fábrica de ladrillos; lo anterior, a través de la identificación de situaciones negativas, a partir de la aplicación de la herramienta de árbol de problemas. A través del análisis realizado, se estableció que la dificultad central de la organización está relacionada con la poca incursión en el mercado regional y local. Por esta razón, el objetivo planteado en la investigación incluyó el diseño de un plan estratégico para esta empresa manufacturera, ubicada en Colombia, que abarque el periodo 2021-2025. Lo anterior, con el objetivo de que esta pueda ampliar sus mercados más allá de lo local y de lo regional, a través de la implementación de estrategias a corto y a mediano plazo, las cuales se representarán en un Cuadro de Mando Integral. El estudio se desarrolló con enfoque mixto y con un alcance descriptivo - explicativo, empleando la metodología de investigación de caso de estudio. Como resultado final, fue posible identificar los factores de cambio que se consideran indispensables para desarrollar estrategias, las que su vez, fueron implementadas con gran satisfacción, asunto que permitió dar solución a la problemática evidenciada.

Palabras clave: Planeación estratégica, mercado, MIC MAC.

\section{Abstract}

This paper analyzes the factors of change in the development of strategies in a brick factory company through the identification of negative situations with the application of the Problem Tree tool in which it was established that the central difficulty of the organization is the little incursion in the regional and local market. For this reason, the objective of the research included the design of a strategic plan for this manufacturing company located in Colombia, for the period 2021-2025, in order to expand markets outside the regional and local ones, through the implementation of short and medium term strategies represented in a Balanced Scorecard. The study was developed with a mixed approach and a descriptive-explanatory scope, using the case study research methodology. As a final result, it was possible to identify the indispensable change factors to develop

Rey-Sánchez, J.C.; García-Delgado, B.A.; Sepúlveda-Mujica, Y. y García-Méndez, S. (2021). Planeación estratégica: caso de estudio aplicado a una fábrica de ladrillos en Colombia. Revista FACCEA, Universidad de la Amazonia, Vol. 11(1), 138-149. https://doi.org/10.47847/faccea.v11n2a3 
strategies, which in turn were implemented with great satisfaction, which allowed the solution to the evidenced problem.

Key words: strategic planning, market, MIC MAC.

\section{INTRODUCCIÓN}

En el departamento de Santander, el sector ladrillero tuvo un incremento significativo desde el año 2011; sin embargo, a partir del año 2016 ha presentado un decrecimiento importante. Según el informe presentado por Compite 360 (2020), después del año 2011 y hasta el 2020, se constituyeron 16 empresas, las cuales representaban el $48.5 \%$ de la totalidad de las organizaciones del sector, desde el año 1990. No obstante, en el periodo comprendido entre los años 2016 y 2019, se cancelaron en total 15 de estas organizaciones, las cuales se dedicaban a actividades de alfarería. Lo anterior, indica la importancia de determinar el perfil estratégico de las empresas de dicho sector, con el fin de establecer un plan estratégico, que conduzca a mejorar la competitividad del mismo. Este tipo de perfilamiento estratégico consta de un análisis, para evaluar el entorno, identificando algunas oportunidades y amenazas. Gómez \& Castellanos (1996) afirman que el perfil estratégico consta de un esquema, el cual permite observar la posición en la cual se encuentra la empresa, respecto de sus factores críticos; por lo tanto, se deben elegir detalladamente los factores internos y externos más importantes, asignándoles una valoración que va desde "muy negativa" hasta "muy positivo".

En este sentido, en el presente artículo se desarrolla el plan estratégico para una fábrica de ladrillo, a fin de que esta organización amplíe la oportunidad de nuevos mercados locales y regionales. Para ello, han sido aprovechadas las fortalezas centradas en su infraestructura, tanto de maquinaria, como de tecnología, así como el amplio conocimiento que ésta ha adquirido en el sector, gracias a su gran trayectoria en el mismo. Asu vez, este plan se desarrolla realizando una investigación exhaustiva, en la cual se puede identificar la problemática central que presenta actualmente la organización. Posterior a esto, se detectan las fortalezas que posee la empresa; las debilidades que se han trabajado para convertirse en oportunidades; las amenazas que se han contrarrestado y las oportunidades que no se han aprovechado. De igual manera, se estructuran e implementan nueve líneas estratégicas, mediante la formulación de un plan estratégico. Este proceso ha contribuido, en gran medida, a dar solución a la problemática detectada.

\section{METODOLOGÍA}

La presente investigación se llevó a cabo bajo un enfoque mixto, en tanto este consiste en la recolección y el análisis de datos cualitativos y cuantitativos, que se relacionan entre sí, para darle un resultado más preciso al fenómeno de estudio (Hernández R. \& Mendoza C., 2018). Igualmente, para el estudio se adaptaron dos alcances diferentes, uno descriptivo y otro explicativo, por cuanto se pretende especificar los aspectos que han de ser analizados y explicar de manera razonable su ocurrencia. En tal sentido, por un lado con el alcance 
descriptivo se busca detallar de forma precisa las características y las propiedades de los procesos, así como los objetos o los fenómenos que se quieren someter a un análisis; por otro lado, con el alcance explicativo se pretende comprender por qué sucede un determinado fenómeno y en qué condiciones se manifiesta este (Hernández-R. et al., 2014). Asimismo, para llevar a cabo el proyecto se adaptó la metodología de investigación denominada "Estudio de Caso", basada en un diseño no experimental de corte transversal, desde el cual se utiliza cualquier enfoque de investigación (cualitativa, cuantitativa o mixta) y se realiza un análisis a fondo de la unidad o del caso investigado (individuo, organización); lo anterior, con la finalidad de darle solución al planteamiento del problema, así como para probar hipótesis, apoyándose en investigaciones previamente realizadas (Hernández-Sampieri, 2014).

Para llevar a cabo el proceso metodológico, inicialmente se realizó un árbol de problemas, con el fin de identificar las causas y las consecuencias de las dificultades que tiene la fábrica de ladrillos de Santander, la cual fue objeto de estudio de esta investigación. Posteriormente, se ejecutó un análisis al entorno interno de la organización, mediante herramientas como la lista de chequeo o el listado de preguntas estructuradas en forma de cuestionario, calificadas con una escala de Likert, a partir de la cual se establecieron los siguientes parámetros: (1) No Existe Importancia; (2) Poco Importante; (3) Neutral; (4) Importante y (5) Muy Importante. Estas variables permitieron identificar y contrarrestar las fallas que pudieron surgir al momento de desarrollar cualquier actividad, dentro de una organización (Grajales-Gaviria \& Castellanos-Polo, 2018), así como determinar la cadena de valor. También se efectuó un análisis del entorno general de la organización, usando matriz de análisis PESTEL, la cual permitió identificar las oportunidades y las amenazas que pudieron afectar y beneficiar a la empresa (González-Palacio \& González-Palacio, 2017) (Santos Jiménez, 2014) ; igualmente, se utilizó la matriz de perfil competitivo, la cual facilitó observar la posición en la cual se encontraba la empresa, respecto de sus factores críticos (Gómez \& Castellanos, 1996). También fueron utilizadas las cinco fuerzas competitivas de Porter, como modelo de análisis competitivo, que se usa para determinar la productividad que tiene el sector, en el cual se encuentra ubicada la organización y la oportunidad que tiene este para sobrevivir en el mercado, al cual se dirige (Donawa Torres \& Morales Martínez, 2018)-. Luego, se aplicó la matriz DOFA cuantificada, la cual sirvió para observar las estrategias, la posición y la dirección de una empresa, o para la creación de una idea (Hill \& Westbrook, 1997; Shinno et al., 2006). Finalmente, se estructuraron las líneas estratégicas de la organización, aplicando la herramienta de análisis estructural MICMAC, un método cualitativo que relaciona diferentes elementos de un sistema, con el fin de provocar cambios en el futuro, identificando variables influyentes y dependientes, para analizar su relación con el sistema de la organización (Delgado Martínez \& Pantoja Timarán, 2015) (Perez-Uribe \& Vargas, 2017).

\section{RESULTADOS}

\section{Árbol de problemas}

Mediante la realización de un árbol de problemas se logran identificar las causas y las consecuencias que genera el problema principal que presenta la fábrica de ladrillos, la cual es objeto de este estudio. En tal sentido, se encuentra que a la compañía le falta incursionar en otros mercados, que sean diferentes al regional y al local, asunto que a su vez está relacionado directamente con la inexistencia de una planeación 
estratégica. La primera causa identificada para este fenómeno, tiene que ver con que la línea de producción de la organización es muy limitada; por lo tanto, sólo puede producir una determinada variedad de productos, lo que conduce, en primera instancia, a enfocar la atención en un solo segmento del mercado, el cual actualmente son las ferreterías y constructoras. A su vez, esto causa una alta especialización del trabajo, lo cual se considera inconveniente, debido a que no existen empleados polivalentes, que tengan nuevas ideas, a través de las cuales beneficien a la organización. Igualmente, esta problemática relacionada con la limitación, trae consigo consecuencias que resultan ser de gran impacto para la empresa; entre ellas se encuentran, por ejemplo, el poco reconocimiento, como un factor que pone en riesgo muchos aspectos, como los volúmenes de ventas, los cuales, al no tener un mercado suficiente, se podrían reducir en una cantidad considerable y, por tanto, los índices de rentabilidad se verían afectados, con la reducción de las ventas.

Además, en la organización objeto de estudio la toma de decisiones se lleva a cabo de forma tradicional. Esto se debe a dos problemas específicos; por una parte, al desconocimiento del entorno y, por otra, a la poca comprensión que se tienen en general, sobre la industria y sobre el sector productivo. Lo anterior, ha ocasionado algunos inconvenientes, por ejemplo, la ausencia de proyección y de objetivos organizacionales, o la falta de políticas empresariales; no obstante, si estos problemas no se corrigen, traerán consigo consecuencias más difíciles de reparar, entre las cuales se encuentra el desaprovechamiento de nuevas oportunidades. Por ello, es necesario indicar que lo que aquí se ha presentado es un fenómeno importante, en tanto las situaciones descritas podrían llevar a la empresa a disminuir la cantidad de sus clientes. Por otra parte, también se evidencia la toma de malas decisiones, en tanto existe una ausencia de liderazgo y, a su vez, es evidente una falta de personal que cuente con los conocimientos necesarios en la planta de producción; lo anterior, también puede producir consecuencias negativas como lo son la desorientación que, a su vez, provoca la falta de metas organizacionales y la desmotivación del personal, ocasionando un alto índice de rotación de los colaboradores; valga indicar, que estas situaciones se deben atender, para no interrumpir la integridad de la organización. De igual manera, la delimitación de materias primas es una de las causas por las cuales la organización ofrece una reducida variedad de productos; esto dificulta que diferentes nichos de mercado se interesen por los mismos, limitando a su vez el crecimiento y el desarrollo, ya que no se incursiona en nuevas ideas, afectando así la posibilidad de obtener mayores utilidades. Asimismo, otro de los factores por los cuales se ve afectada la productividad de la empresa tiene que ver con las pausas ocasionadas por el mantenimiento de las máquinas, el cual se realiza en cualquier momento del horario laboral, situación que afecta el flujo de la producción, ocasionado retraso en los pedidos de los clientes; ello, además conduce a una desventaja competitiva.

\section{Análisis del entorno interno de la organización}

En esta etapa se aplican algunas herramientas técnicas, a través de las cuales se recopila información a nivel interno, en todas las áreas de la organización; entre estas herramientas se encuentra la lista de chequeo, realizada por el método de la observación directa y la cadena de valor. En primer lugar, la lista de chequeo se implementa considerando las $5 \mathrm{MQS}$, como variables para todas las áreas funcionales de la organización. Estas variables de estudio están relacionadas con los despilfarros de las empresas y hacen referencia a los siguientes factores: personas (este despilfarro consiste en tener más recurso humano del que en realidad se 
requiere o se necesita); máquinas (hace referencia a la compra de equipos que realmente no se requieren y que no se justifican); materiales (consiste en emplear elementos o piezas en los productos que resultan siendo innecesarios); métodos (relacionado directamente con el tiempo que se está desperdiciando porque no agrega valor al producto); calidad (son los productos que salen de manera defectuosa) y seguridad (hace referencia a los accidentes de trabajo o a las enfermedades laborales) (García-Méndez et al., 2020) (MoralesMéndez \& Silva-Rodríguez, 2020).

La herramienta antes señalada, a su vez permite identificar 30 fortalezas indispensables, que deben ser aprovechadas al máximo, mediante la ejecución de estrategias puntuales. La variable en la que existen más fortalezas es en la maquinaria, lo cual resulta ser de suma importancia al momento de competir, debido a que, en este sector, la implementación de tecnología resulta ser un proceso costoso; además, a que, en la mayoría de las industrias de este tipo, aún existen procesos muy artesanales. Por otra parte, se deben contrarrestar las debilidades, principalmente, en la variable de dirección, ya que en la organización objeto de estudio, la toma de decisiones es muy conservadora y rudimentaria.

También resulta fundamental analizar la cadena de valor de la organización, con el fin de conocer a profundidad su microentorno y así detectar aspectos clave, para ser empleados en la formulación del plan estratégico. Por esta razón, se analiza la Cadena de Valor actual de la empresa; respecto de esta, se encuentra que muestra una infraestructura fuerte, un alto potencial tecnológico, una buena logística de entrada y de salida, y unos métodos adecuados, empleados para la atención y la fidelización de clientes, como lo son los servicios postventa y las ofertas. Con la realización de la cadena de valor se identifican los diferentes procesos que existen en la empresa, tanto los que implican actividades de soporte, como los que implican actividades operacionales. De esta manera, se puede establecer que la organización cuenta con tecnología de punta, lo que la hace más competitiva en el sector; a su vez, posee una infraestructura óptima, lo que le permite adecuar o modificar cualquier tipo de maquinaria. No obstante, la actividad operacional de marketing carece de estrategias de publicidad para su reconocimiento, lo cual limita su alcance hacia nuevos mercados y hacia clientes potenciales, para seguir creciendo en el sector.

\section{Análisis del entorno general de la organización}

En esta etapa se realiza un diagnóstico con ayuda de herramientas como la matriz de análisis PESTEL, la matriz de perfil competitivo y las cinco fuerzas competitivas de Porter. Con la información que surge a partir del uso de estas herramientas se identifican oportunidades que pueden ser aprovechadas, así como amenazas que afectan el rendimiento de la empresa, frente a la competencia. Por lo anterior, se desarrollan estrategias que se pueden emplear, con el objetivo de explotar al máximo las oportunidades y de minimizar el riesgo que pueden representar las amenazas. En primera instancia, para el análisis externo se implementa la herramienta PESTEL, mediante la investigación de fuentes secundarias tales como revistas, periódicos, páginas web, entidades gubernamentales, opiniones de expertas en el sector ladrillero y de la construcción. Con la información recopilada se estructura una matriz, a partir de la cual se identifican aquellos factores externos que la empresa debe atender, con el fin de explotar las oportunidades y de debilitar las amenazas. Dentro del factor económico se detectan aspectos importantes como el PIB, la inflación y el desempleo. Con 
respecto al factor Político- Legal se encuentra información relevante sobre políticas públicas, reformas, políticas ambientales y de seguridad y salud en el trabajo. En cuanto al factor social se evidencian datos sustanciales, como el crecimiento del sector de la construcción, programas de vivienda ofrecidos por el gobierno y otros beneficios económicos a las empresas; también se evidencia el nivel de informalidad que existe actualmente en las empresas de este sector. En el entorno tecnológico, se conoce que la implementación de tecnología, por parte de los competidores, ha aumentado considerablemente. Finalmente, respecto del entorno ecológico se logra conocer información crucial, como la influencia negativa de los cambios climáticos y las condiciones que debe tener la empresa para operar, en relación con el cuidado del medio ambiente.

Igualmente, la herramienta de las 5 fuerzas de Porter permite analizar la empresa desde cinco puntos de vista, los cuales se entienden fundamentales para el desarrollo y para la competitividad de aquella. (1) El primero consiste en la amenaza de nuevos competidores; allí se detectó que existe un porcentaje considerable de ladrilleras que ingresan latentemente al sector de manera informal. Según la Asociación Nacional de Empresarios en Colombia (ANDI, 2019), el 25\% de las toneladas de ladrillos en Colombia es producido por la gran industria, en tan solo el $3 \%$ de los hornos, mientras que el $75 \%$ restante se produce en el $97 \%$ de hornos; esto corrobora que existe una alta informalidad en el sector. (2) Para el segundo punto, se encuentra que de igual manera se debe evaluar a los productos sustitutos, como variable importante, ya que si existen más productos sustitutos disponibles, los clientes podrán renunciar a adquirir un producto con el cual no están satisfechos, ya sea por precio, por calidad, etc. (Donawa Torres \& Morales Martínez, 2018). Sobre este factor se detecta que existe una alta amenaza para la empresa, debido a que hay diversos artículos que satisfacen las mismas necesidades que los ladrillos de arcilla; entre estos se encuentran los ladrillos prefabricados con plástico, ladrillo de cemento, la madera, el bambú, entre otros. (3) Respecto del tercer punto, se detecta que existe un alto poder de negociación con los proveedores, aunque también se conoce que no se cuenta con un catálogo amplio de ellos. La relación con los proveedores es uno de los factores más importantes para que el negocio funcione; sin el apoyo de estos se pueden generar pérdidas (Varela Neira et al., 2009). (4) Con respecto al cuarto punto, adicionalmente se detecta que el poder de negociación con los clientes es alto, y que siempre se busca un mutuo beneficio entre ambas partes. Para impulsar la negociación con los clientes se desarrollan estrategias que buscan establecer una conversación colaborativa, profesional y respetuosa, además del ofrecimiento de posibles soluciones para los clientes, antes de realizar la compra. (5) Finalmente, con respecto al quinto punto se encuentra que existe una gran rivalidad entre competidores, debido a que todos están optando por implementar tecnología, a la vez que están pensando en la sostenibilidad ambiental. De acuerdo con Donawa Torres \& Morales Martínez (2018)-, se deben establecer estrategias que posicionen el producto en el mercado, identificando las posibles amenazas que se presentan, para así afrontarlas con profesionalismo. Por lo tanto, implementar una estrategia de marketing sería de gran ayuda, para ofrecer un producto que se diferencie de la competencia, además de mostrarse su proceso productivo, en una campaña publicitaria, para que el cliente conozca el origen del producto como tal, inclinándose por el mejor producto y servicio que se ofrece. 


\begin{abstract}
Matriz de perfil estratégico
Después de aplicar las diferentes herramientas que permiten la recolección de información a nivel interno y externo sobre la organización objeto de estudio, ha sido posible conocer las fortalezas, las oportunidades, las debilidades y las amenazas, que serán necesarias para establecer las líneas estratégicas en la etapa siguiente del proceso. Es importante resaltar que, debido a que se presenta una cantidad considerable de aspectos, se ha hecho necesario emplear la matriz de perfil estratégico, para unificar la cantidad de factores y posteriormente construir la matriz DOFA. Este proceso se realiza con el fin de decantar aquellos aspectos más relevantes que la empresa debe atacar; para explotar los que le contribuyen y para contrarrestar los que no. De igual manera, es posible afirmar que se escogen 10 aspectos considerables de cada entorno, los cuales serán evaluados, con el fin de plantear líneas estratégicas a seguir. Allí se detectan resultados muy satisfactorios; desde conocer que la empresa implementa tecnologías de punta, lo cual le permite tener procesos óptimos y eficientes, hasta saber que actualmente no existe disposición por parte de los hogares para comprar vivienda, por lo cual el sector se ve afectado considerablemente.
\end{abstract}

\title{
Desarrollo de la matriz DOFA cuantificada
}

Con la ayuda y desarrollo de la matriz de perfil estratégico se pudo facilitar el proceso de ejecución de la matriz de análisis DOFA, en la cual se identificaron fortalezas, debilidades, oportunidades y amenazas, que contribuyeron con el progreso de la organización.

Fortalezas: personal capacitado para nuevos retos (empleados polivalentes); implementación de tecnología que permite procesos más óptimos y eficientes; aprovechamiento de la capacidad instalada de los equipos; alto poder negociador con proveedores locales y nacionales; conocimiento detallado de los pronósticos de demanda; procedimiento estructurado en las funciones de logística de abastecimiento y distribución; estándares de calidad actuales que cumplen con los exigidos en el mercado regional y local; personal encargado (1) de supervisar el cumplimiento del uso de los EPP; pólizas de seguros que respaldan la organización en caso de algún inconveniente; infraestructura actual adecuada y apta para operar y para garantiza la integridad del personal.

Debilidades: no existe recurso humano adecuado para desarrollar actividades específicas en cada área; no se realizan mantenimientos preventivos en las fechas correspondientes; no existe un catálogo amplio de proveedores; la cadena de suministro de la organización no está claramente estructurada y no proporciona efectividad para lograr rapidez en la adquisición de materias primas; la empresa no posee buenas relaciones con operadores logísticos (2PL ,3PL, 4PL, 5PL); los turnos laborales no son adecuados para aprovechar la capacidad instalada de la planta y gran parte del mercado; los objetivos empresariales no son claros ni alcanzables en el tiempo; la comunicación interna es deficiente en toda la organización; no existe un manual de funciones, ni políticas establecidas que garanticen la calidad en los productos y en los procesos; no existe la implementación del Sistema de Seguridad y Salud en el Trabajo.

Oportunidades: garantiza política y legalmente la sostenibilidad, mediante certificaciones otorgadas por entidades gubernamentales; el gremio de la construcción ha propuesto garantizar la suficiencia de 
subsidios, para los programas de Vivienda de Interés Social, los cuales son la base para reactivar la demanda de vivienda y para lograr que los colombianos encuentren todas las oportunidades de la inversión inmobiliaria; el $91 \%$ (2.379 proyectos) de los proyectos de construcción ya retomó actividades, lo que ha garantizado el empleo para cerca de 600.000 trabajadores; el sector de la construcción crecerá a un promedio de $5 \%$, ubicándose como uno de los sectores que conducirán el crecimiento del país; actualmente, el Gobierno Nacional de Colombia tiene cuatro programas de vivienda, Casa Ya, Casa Ahorro, programa casas gratis y subsidios de créditos, mostrando un fuerte apoyo a este sector; de acuerdo con los cálculos de Asogravas, se estima que la producción de materiales de construcción pase de 160 millones de toneladas a 320 millones en 2025; el auge en el sector de la construcción de vivienda y de obras de infraestructura, incrementará la demanda de materiales de construcción; el crecimiento de la clase media y el mayor poder de compra de los hogares, favorecerá la demanda de vivienda; políticas y licencias ambientales para la explotación de minas e implementación de un plan de manejo ambiental para la organización.

Amenazas: en el segundo trimestre de 2020 el PIB de Colombia cayó 15,7\%; la disposición de los hogares para comprar vivienda en Colombia es de -62,63\% en total, en Bucaramanga se presentó la cifra más baja (-78,5\%);la economía colombiana podría decrecer entre 2,7\% y 7,9\% en el 2020; Colombia lidera el ranking con un contundente porcentaje de desempleo, la tasa histórica es de 21,4\%; hay una escasez de personal calificado para los requerimientos de instalación, operación y mantenimiento de la maquinaria implementada; implementación de tecnología entre los competidores; altos costos en la modificación o en la implementación de tecnología; el $25 \%$ de las toneladas de ladrillos en Colombia es producido por la gran industria, en tan solo el $3 \%$ de los hornos, mientras que el $75 \%$ restante se produce en el $97 \%$ de los hornos, lo que corrobora la alta informalidad; los cambios climáticos producen derrumbes que dificultan el acceso y la salida de la organización; las lluvias dificultan la explotación de las minas de arcilla.

De acuerdo con los resultados expuestos se puede afirmar que la organización presenta fuertes debilidades. Entre estas se destaca la ausencia de un manual de funciones, por lo cual los empleados no saben a quién obedecer; de igual manera, se detectó que la empresa posee un número reducido de proveedores, y que, además, no cuenta con un personal que desarrolle actividades específicas de una sola área. Por otra parte, también se detectaron fortalezas, las que a su vez ofrecen ventajas competitivas frente al rival; la más importante de estas, es que todos sus procesos productivos son totalmente automatizados.

A su vez, para el proceso de cuantificación de la matriz DOFA se formularon algunas preguntas, las cuales permitieron enlazar los diferentes factores internos y externos. Entre estas se encuentra la influencia entre la fortaleza y la oportunidad, cuestionándose en qué medida la fortaleza permite aprovechar la oportunidad, o en qué medida la fortaleza permite contrarrestar la amenaza. De igual manera, se analizaron los aspectos negativos preguntándose, en qué medida, la corrección de la debilidad permite aprovechar la oportunidad, 0 en qué medida, la corrección de la debilidad permite contrarrestar la amenaza. Cabe mencionar que esta evaluación se realizó bajo la escala de 1 a 5 (donde 1 era un valor muy bajo, lo quiere decir que no podía ser aprovechada; 2 un valor bajo; 3 medianamente indiferente; 4 valor alto y 5 como un valor muy alto, en el cual 
se puede aprovechar la fortaleza o debilidad planteada), con la colaboración de expertos, quienes proporcionaron sus conocimientos para tener un proceso real y con alta contribución en los resultados de la investigación; los resultados de la evaluación se presentan en la figura 1.

Una vez realizada la cuantificación de la Matriz DOFA se pudo conocer una serie de fortalezas, de oportunidades, de debilidades y de amenazas, que resultan las más relevantes y las cuales se tuvieron en cuenta para dar paso a la siguiente etapa de desarrollo. Dentro de los factores internos de la organización se destacan la falta de mantenimientos preventivos; las carencias de relaciones con operadores logísticos externos; la inexistencia de las funciones que se deberían asignar a cada colaborador; la falta de implantación de normas fundamentales para el funcionamiento y la escasez de objetivos que contribuyen al desarrollo de la organización.

\section{Plan estratégico}

En esta fase se estructuraron las líneas estratégicas, aplicando la herramienta de análisis estructural MIC-MAC, lo cual se realizó mediante una reunión de expertos en el sector ladrillero y de construcción (Método Delphi). Este proceso permitió conocer las líneas que se deben atender de forma inmediata; aquellas que son muy importantes y que comprenden acciones de carácter transformador $y$, finalmente, aquellas que definitivamente si no se consideran, no influirían muy significativamente. Para ello, en primer lugar se realizó un proceso de evaluación a los factores de cambio considerados, gracias a las fortalezas y debilidades detectadas, mediante la matriz DOFA cuantificada; para esto, se empleó el método Delphi y la siguiente pregunta: ¿Qué incidencia tiene el factor de cambio "X" con los demás factores para favorecer procesos de emprendimiento en el sector y generar emprendimiento en la región?. Después de realizado ese ejercicio, se detectó que existen factores de cambio que presentan dependencia significativa, respecto de los demás; entre éstos, se encuentra la conservación de la calidad en los productos, para que así sea posible incrementar la demanda y la participación en el mercado; también se encuentra la mejora de la capacidad instalada de los equipos; el aprovechamiento de la capacidad instalada de los equipos y la estructuración de objetivos empresariales claros y alcanzables en el tiempo. Se entiende a su vez que esta dependencia es clave, porque llevará a que los factores mencionados se interrelacionen entre sí y se conviertan en líneas estratégicas para alcanzar el éxito. Cabe mencionar que los demás factores de cambio no son menos importantes, aunque simplemente no necesitan una dependencia muy alta, para convertirse en una línea estratégica fundamental, para direccionar la organización.

Por otra parte, para el reconocimiento y la ubicación de cada uno de los factores de cambio se calculó el promedio de los dos ejes $(X, Y)$, los cuales determinarán la posición de los factores en un plano cartesiano, en el cual, el promedio calculado se divide en cuatro zonas (zona de poder, zona de conflicto, zona de salida y zona de independencia), clasificándolo en los ejes que representan ( $X=$ Dependencia, $Y=$ Motricidad); lo anterior, con el fin de tomar la decisión acerca cuál es el factor en el que hay que concentrar los mayores esfuerzos; de igual manera, para convertirlo en un estrategia para la organización. Siguiendo esta misma línea, después de finalizar el proceso de evaluación se encontró que existen diez factores clave, por encima de la pendiente, para trabajar en las líneas estratégicas; de entre estos, tres se encuentran en la zona de poder, es decir, estos son sobre los que hay que actuar de inmediato, debido a que tienen alta motricidad y baja dependencia. También, se pudo evidenciar que cinco de los factores detectados se ubicaron en la zona de conflicto; estos tienen alta motricidad y alta dependencia y, además, son de un nivel clave y deben atenderse, para que no sean convertidos en cuellos de botella. Finalmente, se detectó que dos de estos factores se encuentran en la zona de autonomía, es decir, que son independientes y que tienen poca motricidad, pero que no dejan de ser fundamentales. 
Figura 1.

Matriz DOFA cuantificada.

\begin{tabular}{|c|c|c|c|c|c|c|c|c|c|c|c|c|c|c|c|c|c|c|c|c|c|c|}
\hline & $\mathrm{O} 2$ & 05 & 07 & 010 & O11 & 012 & $\mathrm{O} 14$ & 019 & 021 & $\mathrm{O} 23$ & $\mathrm{Al}$ & A4 & A7 & $\mathrm{A} 10$ & A12 & $\mathrm{A} 18$ & $\mathrm{~A} 19$ & A21 & $\mathrm{A} 22$ & A25 & & \\
\hline $\mathrm{Fl}$ & 3 & 4 & 1 & 3 & 4 & 5 & 5 & 3 & 1 & 1 & 4 & 3 & 3 & 4 & 5 & 5 & 2 & 3 & 2 & 2 & & 63 \\
\hline F5 & 5 & 4 & 4 & 4 & 5 & 3 & 5 & 4 & 1 & 1 & 4 & 1 & 2 & 5 & 2 & 4 & 2 & 5 & 5 & 5 & & 71 \\
\hline F6 & 1 & 4 & 2 & 3 & 4 & 4 & 5 & 5 & 1 & 1 & 4 & 1 & 1 & 4 & 2 & 4 & 1 & 5 & 1 & 2 & & 55 \\
\hline $\mathrm{F} 12$ & 2 & 4 & 4 & 3 & 5 & 2 & 4 & 3 & 1 & 1 & 3 & 1 & 1 & 2 & 3 & 5 & 5 & 4 & 1 & 1 & $\exists$ & 55 \\
\hline F17 & 1 & 5 & 2 & 4 & 5 & 3 & 4 & 4 & 1 & 1 & 2 & 1 & 1 & 3 & 2 & 3 & 1 & 4 & 1 & 1 & $\exists$ & 49 \\
\hline $\mathrm{F} 20$ & 1 & 2 & 2 & 4 & 2 & 2 & 4 & 4 & 1 & 1 & 1 & 2 & 1 & 1 & 2 & 3 & 4 & 4 & 5 & 2 & $\exists$ & 48 \\
\hline $\mathrm{F} 23$ & 5 & 2 & 2 & 2 & 4 & 2 & 3 & 2 & 1 & 1 & 4 & 4 & 2 & 1 & 2 & 5 & 1 & 5 & 2 & 1 & $\underline{\Xi}$ & 51 \\
\hline $\mathrm{F} 27$ & 1 & 1 & 4 & 1 & 1 & 1 & 2 & 2 & 1 & 1 & 1 & 2 & 2 & 1 & 3 & 1 & 1 & 2 & 2 & 2 & $T$ & 32 \\
\hline $\mathrm{F} 28$ & 1 & 1 & 1 & 1 & 1 & 1 & 1 & 1 & 3 & 1 & 1 & 2 & 2 & 1 & 2 & 2 & 4 & 2 & 5 & 5 & $\stackrel{\rho}{\sim}$ & 38 \\
\hline $\mathrm{F} 29$ & 2 & 4 & 5 & 1 & 3 & 2 & 3 & 3 & 1 & 1 & 3 & 1 & 2 & 4 & 1 & 4 & 5 & 5 & 3 & 3 & 8 & 56 \\
\hline D1 & 3 & 1 & 2 & 2 & 3 & 3 & 4 & 4 & 1 & 1 & 1 & 2 & 1 & 5 & 4 & 1 & 2 & 3 & 2 & 1 & 정 & 46 \\
\hline D7 & 1 & 4 & 4 & 1 & 4 & 3 & 4 & 4 & 1 & 1 & 1 & 2 & 2 & 4 & 4 & 1 & 5 & 2 & 2 & 2 & $>$ & 52 \\
\hline D10 & 5 & 4 & 3 & 3 & 5 & 4 & 5 & 5 & 1 & 1 & 2 & 2 & 2 & 5 & 1 & 2 & 5 & 4 & 2 & 1 & $\prec$ & 62 \\
\hline D12 & 1 & 5 & 3 & 4 & 2 & 4 & 5 & 5 & 1 & 1 & 1 & 2 & 2 & 3 & 1 & 3 & 2 & 3 & 1 & 4 & 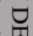 & 53 \\
\hline D14 & 2 & 5 & 3 & 4 & 5 & 5 & 5 & 5 & 1 & 1 & 1 & 1 & 1 & 5 & 1 & 3 & 2 & 4 & 5 & 5 & $\underline{\sigma}$ & 64 \\
\hline D16 & 3 & 4 & 4 & 2 & 4 & 2 & 4 & 4 & 1 & 1 & 2 & 2 & 1 & 4 & 1 & 3 & 1 & 3 & 2 & 1 & $\Xi$ & 49 \\
\hline D20 & 1 & 4 & 2 & 2 & 4 & 4 & 5 & 5 & 1 & 1 & 4 & 3 & 2 & 2 & 1 & 1 & 4 & 4 & 2 & 2 & $\vec{z}$ & 54 \\
\hline D23 & 4 & 5 & 4 & 3 & 4 & 3 & 5 & 5 & 5 & 5 & 1 & 2 & 2 & 2 & 4 & 4 & 4 & 4 & 2 & 1 & (1) & 69 \\
\hline $\mathrm{D} 26$ & 3 & 2 & 3 & 2 & 3 & 5 & 3 & 3 & 5 & 4 & 2 & 3 & 2 & 4 & 1 & 3 & 2 & 2 & 3 & 2 & & 57 \\
\hline D32 & 5 & 3 & 1 & 1 & 4 & 1 & 5 & 5 & 2 & 2 & 1 & 2 & 1 & 2 & 3 & 3 & 3 & 5 & 2 & 2 & & 53 \\
\hline \multicolumn{23}{|c|}{ APROVECHAMIENTO DE LA OPORTUNIDAD O AMENAZA } \\
\hline
\end{tabular}

Una vez realizado ese proceso se pudo concluir que se consideraron nueve líneas, para trabajar en el plan estratégico que se presentó a la organización. Así mismo, desde estas líneas se pretendió abarcar y mejorar la organización, a partir de los nueve puntos que se relacionan a continuación:

$\checkmark$ Planificar el mantenimiento preventivo para la maquinaria de la organización, a partir de lo cual se especifiquen, tanto los aspectos clave, como las actividades, los periodos, la frecuencia y demás aspectos que permitan llevar un control bien estructurado de este proceso.

$\checkmark$ Estructurar un portafolio de proveedores locales y nacionales, que permita combatir cualquier contingencia que pueda presentarse con el proveedor actual, para así garantizar el abastecimiento de los insumos que son necesarios en el proceso productivo.

$\checkmark$ Organizar la cadena de suministros de la empresa, teniendo claridad en cada uno de los procesos, para así enfocarse en las actividades de cada uno de ellos, garantizando la efectividad del proceso productivo.

$\checkmark$ Desarrollar una red de comunicación interna, con el fin de facilitar la comunicación, y en la cual se integren todas las áreas y se trabaje bajo un mismo propósito, unificando los esfuerzos, para así contribuir a la mejora continua de la empresa.

$\checkmark$ Realizar una aproximación entre la empresa y un operador logístico; para este proceso se propone una empresa de transporte, con el fin de que la organización considere la posibilidad de tercerizar alguno de sus procesos.

$\checkmark$ Implementar el Sistema de Seguridad y Salud en el Trabajo.

$\checkmark$ Establecer una planeación estratégica en la organización, con el fin de promover un enfoque claro hacia el futuro, para alcanzar los propósitos fijados.

$\checkmark$ Crear un manual de funciones para los cargos de la empresa.

$\checkmark$ Identificar despilfarros, con el fin de proponer acciones de mejora para identificarlos, corregirlos o eliminarlos. 


\section{CONCLUSIONES}

En el diagnóstico interno y externo realizado como primera fase del proceso se determinó que, entre las debilidades más relevantes de la fábrica de ladrillos, la cual fue objeto de este estudio, se encontraba la inexistencia de objetivos claros y alcanzables en el tiempo; también se encontró la ausencia de un manual de funciones, a su vez que una deficiente comunicación interna. Igualmente se determinó la ausencia de un control de mantenimientos a la maquinaria y una falta de claridad en la cadena de suministros. De igual manera, se detectaron aspectos positivos y negativos a nivel externo, entre los que se destacan las oportunidades para adquirir viviendas otorgadas por el Gobierno Nacional Colombiano y el crecimiento del sector de la construcción en el país. También se detectaron amenazas contundentes que se deben tener muy en cuenta; entre ellas, la presencia de una alta cifra de empresas informales que participan en este sector.

El plan estratégico diseñado en la segunda etapa permitió direccionar la empresa hacia un futuro que contribuyera a alcanzar la mega organizacional; dicho plan se realizó mediante la construcción de nueve líneas estratégicas, las cuales estaban ligadas a las debilidades y a las fortalezas detectadas, en la etapa de diagnóstico. Entre ellas se encuentra la formulación de un programa de mantenimiento; la implementación de una red social interna; la estructuración de objetivos estratégicos; la construcción del manual de funciones; la identificación de despilfarros, entre otras acciones. Gracias a esto, se identificó que la empresa carecía de los lineamientos fundamentales para su crecimiento y desarrollo, como lo son los valores corporativos, la MEGAorganizacional y los objetivos estratégicos.

Cabe resaltar que la empresa ladrillera, objeto de estudio, podrá aprovechar diferentes oportunidades, como lo son el crecimiento del sector de la construcción a nivel nacional y los diferentes medios que el gobierno está otorgando para comprar vivienda en Colombia; lo anterior, a su vez la prepara para no verse afectada por las amenazas que la circundan, como lo es, por ejemplo, la alta informalidad que existe principalmente en este sector. También es posible concluir que, a nivel interno, el plan estratégico es un punto clave para expandir su mercado, pues, con el conocimiento claro de la meta que se quiere lograr a futuro (MEGA), con el planteamiento de objetivos estratégicos claros y alcanzables, con la óptima comunicación interna, con la mejora y el sostenimiento de los altos estándares de calidad y con la implementación de altas tecnologías de punta, que garanticen procesos eficientes y de alta calidad, es posible lograr su amplificación, en tanto la empresa tiene todo el potencial para abarcar otros mercados, garantizando el cumplimiento, la calidad y la capacidad para enfrentarse y ser reconocida, no solo a nivel regional y local, sino también a nivel nacional.

Finalmente, para futuras investigaciones se recomienda replicar este análisis, en empresas de otros sectores económicos, con el fin de realizar comparaciones y relaciones. Además, se sugiere realizar un seguimiento constante al plan estratégico establecido, revisando avances, estado y consecuencias de las actividades.

\section{REFERENCIASBIBLIOGRÁFICAS}

ANDI. (2019). Ladrilleras de Colombia, unidas en nuevo Comité de la ANDI. ANDI. http://www.andi.com.co/Home/Noticia/15597-ladrilleras-de-colombia-unidas-en-nuevo

Compite 360. (2020). Registro Mercantil de las Cámaras de Comercio aliadas. Compite 360. http://www.compite360.com/portal/ 
Delgado, M., A., M. \& Pantoja, T., F. (2015). Structural analysis for the identification of key variables in the Ruta del Oro, Nariño Colombia. DYNA, 82(191), 27-33. https://doi.org/10.15446/dyna.v82n191.45532

Donawa T., Z. A., \& Morales M., E., C. (2018). Fuerzas competitivas que moldean la estrategia en la gerencia del sector mipyme del distrito de Santa Marta -Magdalena, Colombia-. Revista Escuela de Administración de Negocios, 84. https://doi.org/10.21158/01208160.n84.2018.1919

García-M., S., Parra, L., M. Á. \& Garcés, L., T., P. (2020). Capítulo 17. Diseño de un plan estratégico de turismo en el municipio de Guadalupe (Santander). Economía Solidaria, 241.

Gómez, F., G., \& Castellanos, R., M. M. (1996). Perfil estratégico y análisis económico-financiero: Aplicación a cuatro grandes empresas españolas. Administrando Para La Sociedad, 443-452.

González, P., L., \& González, P., M. (2017). Gestión estratégica de la Comunidad Colombiana de Cómputo Avanzado mediante análisis DOFA y cocreación. Ingeniare. Revista Chilena de Ingeniería, 25(3). https://doi.org/10.4067/S0718-33052017000300464

Grajales-G, D. A., \& Castellanos-P, O. C. (2018). Evaluación del control interno en el proceso de tesorería de las pequeñas y medianas empresas de Medellín. Revista CEA, 4(7). https://doi.org/10.22430/24223182.760

Hernández R., Fernández C., \& Baptista P. (2014). Metodología de la investigación. Mc Graw-Hill.

Hernández, R., \& Mendoza, C. (2018). Metodología de la investigación las rutas cualitativas, cuantitativa y mixta. Mc Graw-Hill.

Hernández, S., R. (2014). Metodología de la investigación. McGraw Hill Education.

Hill, T., \& Westbrook, R. (1997). SWOT analysis: It's time for a product recall. Long Range Planning, 30(1), 46-52. https://doi.org/10.1016/S0024-6301(96)00095-7

Morales-M, J -D., \& Silva-Rodriguez, R. (2020). The Use of Lean Manufacturing Tools to Improve the Production of Automobile Parts. En Akdogan, Anil \& Serdar, Ali. Mass Production Processes. IntechOpen. https://doi.org/10.5772/intechopen.88470

Perez-U, R., I., \& Vargas, H. A. (2017). El uso del método MICMAC, para la definición de procesos de intervención en las organizaciones. Ciencia y Poder Aéreo, 11(1). https://doi.org/10.18667/cienciaypoderaereo.156

Santos, J., N. (2014). Indicadores de Gestión Empresarial. Industrial Data, 3(2). https://doi.org/10.15381/idata.v3i2.6668

Shinno, H., Yoshioka, H., Marpaung, S., \& Hachiga, S. (2006). Quantitative SWOT analysis on global competitiveness of machine tool industry. Journal of Engineering Design, 17(3), 251-258. https://doi.org/10.1080/09544820500275180

Varela, N., C., Vázquez, C., R., \& Iglesias Argüelles, V. (2009). Comportamiento de abandono de la relación de un cliente con la empresa en un contexto de fallo y recuperación del servicio. Cuadernos de Economía y Dirección de La Empresa, 12(40), 143-169. https://doi.org/10.1016/S1138-5758(09)70045-3 